\title{
ADENOMATOID TUMOR OF TAIL OF EPIDIDYMIS - A CASE REPORT
}

Vishwanath T. Thimmaiah ${ }^{1}$

\section{HOW TO CITE THIS ARTICLE:}

Vishwanath T Thimmaiah. "Adenomatoid tumor of tail of epididymis - a case report". Journal of Evolution of Medical and Dental Sciences 2013; Vol2, Issue 32, August 12; Page: 6061-6065.

ABSTRACT: Paratesticular tumours are rare but generally benign neoplasms, of mesothelial in origin. Adenomatoid tumours of epididymis are uncommon and accounts for $30 \%$ of all paratesticular neoplasms. A case of Adenomatoid tumor arising from tail of right epididymis is presented with ultrasound imaging features and review of literature.

KEY WORDS: Adenomatoid, Mesothelial, Ultrasound.

INTRODUCTION: Adenomatoid tumour of epididymis (ATE) was first described by Golden et al as a small mass in scrotal region without any clinical symptoms. These tumours are uncommon with reported incidence of $5 \%$ and comprise $80 \%$ of all benign paratesticular tumours. Most of these tumors are smaller in size with mean diameter less than $2 \mathrm{cms}$ and have preponderance to arise from epididymal tail. Usually ultrasound combined with histopathological examination is diagnostic for majority of the paratesticular tumours followed by surgical excision.

CASE REPORT: A 40 year old male patient presented with painless right scrotal lump progressively increasing in size since 6 months. There was no history of any previous trauma or genitourinary infection. On clinical examination mass was palpated in the region of lower pole of right testis separate from testis, with no tenderness or signs of inflammation. The scrotum wall and left testis was normal. On Ultrasound examination there was a well-defined isoechoic solid mass lesion with a definite hypoechoic capsule in the region of tail of right epididymis. Mass measured $2.6 \times 2.2 \times 2.1$ cms in size with $3.5 \mathrm{~mm}$ capsule. There was no calcification, cystic changes or necrotic changes within the lesion. On colour Doppler, few internal vascularity was noted with low resistive flow. FNAC from the right epididymal mass was inconclusive; following which surgical exploration and excisional biopsy was done. Histopathological examination showed tubules and cords of tumour cells with large intracytoplasmic vacuoles characteristic of adenomatoid tumor.

DISCUSSION: Epididymal tumours form less than 5\% of all the intrascrotal tumours with primary and secondary types. These tumours may be benign or malignant in origin. Epithelial tumours arising from epididymis are of rare primary subtype and comprise mainly adenomatoid, papillary cyst adenoma and leiomyomas (1,2) Primary malignant tumours of epididymis are even are and comprises sarcomas, dysembryonic tumours and malignant epithelial tumours. Adenomatoid tumour usually involve the genitourinary system in both males and females, with fallopian tubes, ovaries and uterus being common areas for females and epididymis, testes, spermatic cord, ejaculatory ducts in males. The main cause for involvement of genital regions is unknown but it may also involve adrenals, lymph nodes, pancreas, mediastinum and pleura $(3,4,5,6)$. ATE can occur in any age group with most common age group being 30 to 40 years with few reported cases in children's and geriatric age groups. Though it can be bilateral left side involvement is more common than right side (7). ATE are usually small in size with average size being $2 \mathrm{cms}$ in diameter with growing up to 
$10 \mathrm{cms}$ in size. Most commonly tail of epididymis is involved, as in our case flowed by head and body regions. After epididymis the most common location for ATE is intratesticular location arising from tunica albuginea. Clinical presentation can vary from being asymptomatic small masses to very painful masses in the scrotum region that can be confused for torsion. ATE are usually painless and most are incidental detected during routine health check due to its smaller size $(8,9,10)$. However bigger sized lesions presents as long standing gradually increasing mass as in our case.

Being noninvasive, safe, easily available, Ultrasonography has become the initial investigation of choice for localizing the epididymal lesions and ATE being a benign disease, can be diagnosed with high sensitivity and specificity. Ultrasound features are well defined predominantly hypoechoic or isoechoic capsulated solid mass lesion arising from tail or head of epididymis. Some lesions may be hyperechoic with a well ill-defined capsule. These well circumscribed solid mass are usually avascular but may demonstrate internal vascularity on colour flow Doppler imaging as in our case. Differential diagnosis for solid mass lesions arising from epididymal tail includes fibrous pseudotumour of epididymis, leiomyoma, atrophied extra testis papillary cystadenomas and angiolipoma. On CT, ATE is well defined solid hypodense mass lesion in the paratesticular region with absent calcification or necrotic changes. MRI helps in diagnosis, as the lesion appears hypointense to surrounding epididymal and testicular parenchyma on T1 and hyperintense on T2 (11). Contrast enhancement is less compared to normal testis on post contrast administration. (12)

Most surgeons prefer surgical exploration of the epididymis in these cases than needle biopsy as it may lead to easy dissemination in cases of malignancy. FNAC is usually performed but may be inconclusive as was in our case.

Histogenesis of ATE is not clear where few pathologists consider it to be a reaction to injury or inflammation, while most believed it to be of mesothelial origin (13). On electron microscopy it can present in a wide spectrum with basic patterns of tubules, cell nests and cords attributable to ATE. Prominent microvilli, desmosomes and tonofilaments if seen are highly suggestive of ATE (14). Mesothelial markers like calretinin and HMBE1 may distinguish this from tumours of non mesothelial origin and thus aide in diagnosis. On histopathology, the excised epididymal tumour was encapsulated with cuboidal to flattened tumour cells in cords and tubules and having large intracytoplasmic vacuoles that is characteristic of adenomatoid tumor. A few other paratesticular tumours have been reported like leiomyomas which have spindle cell as characteristic HPE finding. Papillary cystadenomas may be seen in cases of Von Hippel Lindau disease which may show dilated tubules and cysts as characteristic finding in microscopy. A few other differentials can be hemangiomas, chronic epididymitis, spermatocele and neuro ectodermic tumors on histopathology.

CONCLUSION: ATE is rare benign paratesticular tumours, and should be suspected in any intrascrotal mass lesions. Its separation from a more serious testicular tumour is important as paratesticular tumours have good prognosis compared to testicular tumours. Ultrasonography is very useful in separating paratesticular tumours from that of testicular tumour with high sensitive and specificity for localizing adenomatoid tumor arising from epididymis. Adenomatoid tumours being benign, have no reported cases of recurrence or metastasis after excision. Local excision of the lesion should be the recommended form of treatment which has the added advantage of confirming the ultrasound diagnosis. 


\section{CASE REPORT}

Figure 1a:-Gray scale Ultrasound of scrotum in Longitudinal plane of right testis, epididymal head and tail showing well defined isoechoic mass lesion in the region of tail with a well-defined hypoechoic capsule.

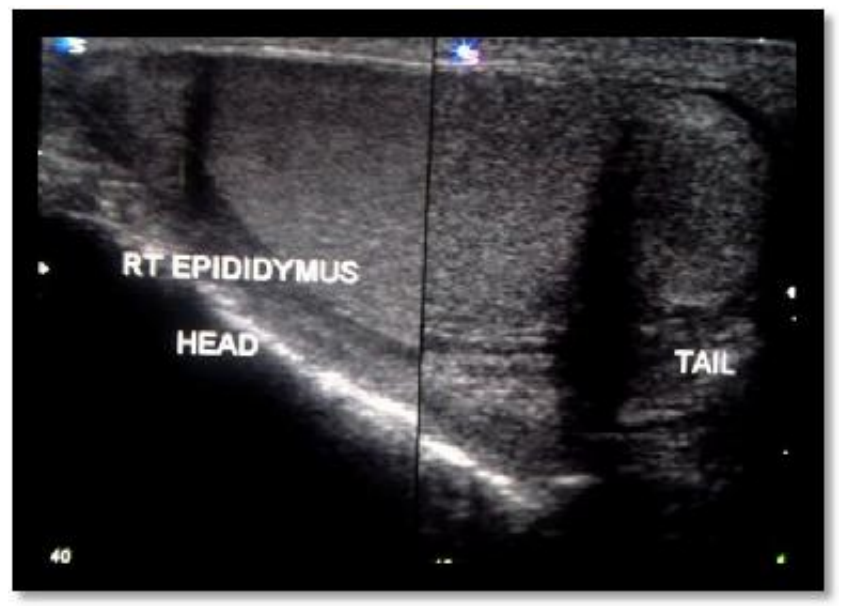

Figure 1b: - Gray scale Ultrasound of scrotum in Transverse plane at the level of lower pole of both testis showing well defined isoechoic solid mass lesion in the region of tail of right epididymis. Left testis and visualized left tail of epididymis are normal.

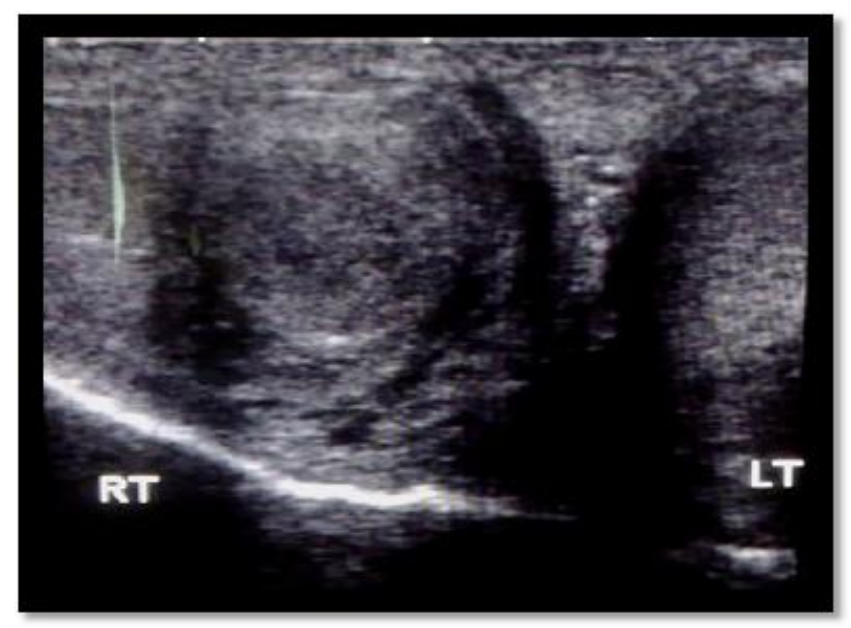


Figure 2:-Colour Doppler of scrotum in Transverse plane at the level of lower pole of right testis showing well defined isoechoic solid mass in the region of tail of right epididymis with internal vascularity.

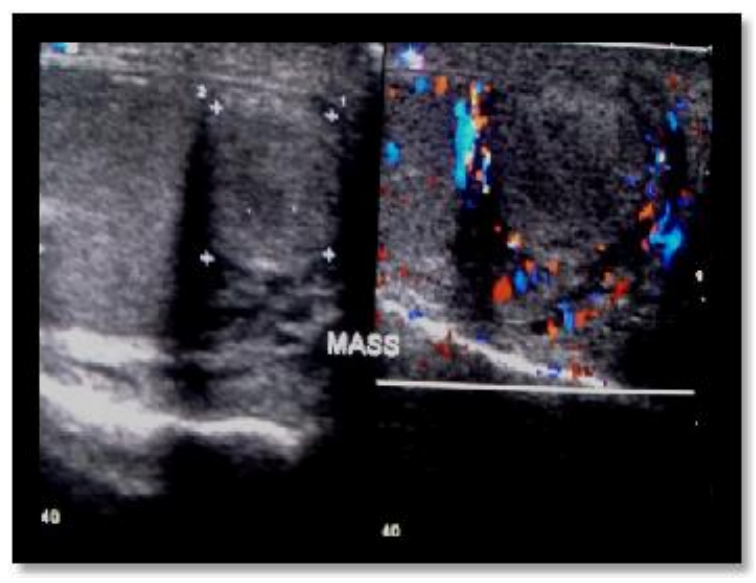

Figure 3:- Histopathology of epididymal mass shows tubules and cords of tumour cells with large intracytoplasmic vacuoles characteristic for adenomatoid tumor.

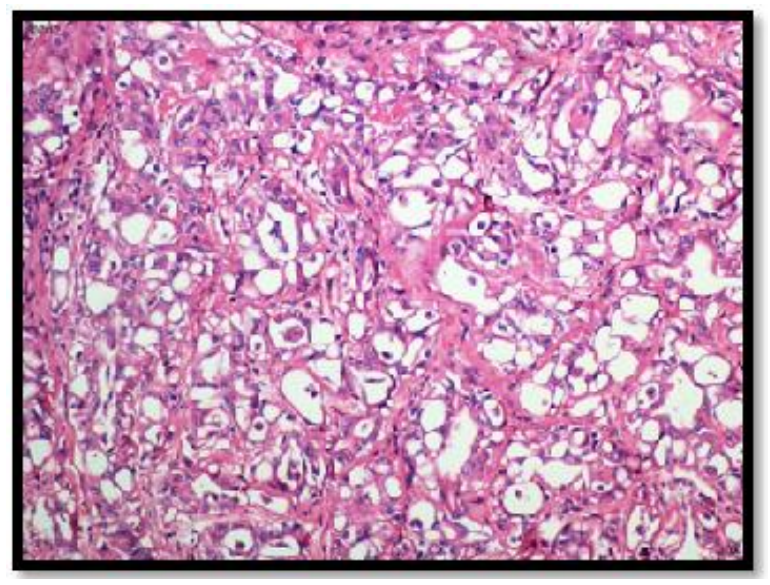

\section{REFERENCES:}

1. Moyano CJL, Giraldez PJ, Sanchez de la Vega J, et al. Adenomatoid tumour of the epididymis. Actas Urol Esp 2007; 31: 417.

2. Samada A, Pereiroa B, Badiola A, Gallegoc C, Zungria E. Adenomatoid tumour of intratesticular localization. Eur Urol 1996; 30: 127-28.

3. Chen KT, Schiff JT. Adenomatoid prostatic tumour. Urology 1983; 21: 88-89.

4. Fan K, Johnson DF. Adenomatoid tumour of ejaculatory duct. Urology 1985; 25: 653-54.

5. Evans CP, Vaccaro JA, Storrs BG, Christ PJ. Suprarenal occurrence of an adenomatoid tumour. J Urol 1988; 139: 348-49.

6. Arcadi J A. Adenomatoid tumour in the tunica albuginea of the testis. J Surg Oncol 1988; 37: 38-39 
7. Srigley JR, Hartwick RWJ. Tumours and cysts of the paratesticular region. Pathol Annu 1990; 25: 51-108.

8. Syed Ali A, Tawfeeq AS, Syed Z HJ, Farnaz H, James S AN. Multimodality imaging of paratesticular neoplasms and their rare mimics. Radio Graphics 2003; 23: 1461-76.

9. Tato RJ, Lema GJ, Cimadevila GA, et al. New report of epididymal Adenomatoid tumour. Infrequent pathology. Acta Urol Esp 1997; 21(5): 502-07.

10. Paula JW, Cornelia MS, Isabell A. Extratesticular Scrotal masses. Radiologic-Pathologic Correlation. Radiographics 2003; 23: 215-40

11. Serrano Frago P, Medrano Llorente P, Borque Fernando A, Pascual Llorente M, Allue López M. Consults by scrotum mass: epididymo lesions]. Actas Urol Esp. 2007; 31(4):420-422.

12. Patel MD, Silva AC. MRI of an adenomatoid tumor of the tunica albuginea. AJR Am J Roentgenol. 2004;182(2):415-417

13. Jackson JR. The histogenesis of the adenomatoid tumour of the genital tract. Cancer 1958; 11: 337-50.

14. Davy CL, Tang CK. Are all adenomatoid tumours adenomatoid mesothelioma. Hum Path 1981; 12(4): 360-69.

\section{AUTHORS:}

1. Vishwanath $\mathrm{T}$ Thimmaiah

\section{PARTICULARS OF CONTRIBUTORS:}

1. Assistant Professor, Department of Radiology, JSS Medical College, JSS University, Mysore, Karnataka, India.

\section{NAME ADRRESS EMAIL ID OF THE CORRESPONDING AUTHOR:}

Dr. Vishwanath. T.T, \#48, HPO \& RMS Layout, Behind RTO East, Shakthi Nagar, Mysore, Karnataka - 570029.

Email - vishurad@gmail.com

Date of Submission: 30/07/2013. Date of Peer Review: 31/07/2013. Date of Acceptance: 06/08/2013. Date of Publishing: 10/08/2013. 